\title{
Editorial
}

\section{The LIFE Trial in 2004}

\author{
Journal of Human Hypertension \\ (2004) 18, 365. doi:10.1038/ \\ sj.jhh.1001746
}

The topic of the treatment of cardiovascular disease, and in particular hypertension, has been very well served with a large number of extremely well conducted studies. Not less than 19 trials have been conducted to investigate whether or not hypertensive patients should receive antihypertensive medication and at what threshold treatment should be started. Only one such trial remains to be completed and that is the HYVET study investigating the value of the reduction of blood pressure in patients over the age of 80 years. Otherwise the era of placebo-controlled trials is over.

The next generation of trials is designed to test whether any one particular class of blood pressure lowering drugs is better than any other at preventing heart attacks and strokes. In general, so far, these trials have been inconclusive and none have shown, convincingly, that the choice of blood pressure lowering drugs matters. It would seem that as long as the blood pressure is reduced any agent will do. Some trials that did appear to show differences in outcome between drugs are difficult to interpret because these differences might have been due to differences in the quality of control of the blood pressure with the various agents compared or an imbalance in the height of the blood pressure at baseline. Clearly, when comparing antihypertensive drugs it is crucial that the severity of hypertension and the quality of the blood pressure control thereafter should be identical so that any differences in outcome can be explained in terms of the drugs used rather than the differences in blood pressure. In this respect the LIFE trial is unique. In this study in 9000 patients, the angiotensin receptor blocker, losartan was compared with the beta-blocker, atenolol. The trial design was refreshingly simple with the dose of both agents being 50-100 mg daily and with hydrochlorothiazide added in as a second agent when necessary. Most important aspect of the LIFE trial is that the blood pressures at entry were practically identical in the two groups as was the quality of control of blood pressure at follow-up. It follows therefore that any differences in outcome cannot be due to differences in blood pressure but must be due instead to particular characteristics of whichever drug comes out best.

The results of this landmark study were truly astounding. Losartan was associated with a statistically significant reduction in primary composite endpoints, mainly explained by a $25 \%$ reduction of first stroke. Furthermore, Losartan patients also had $25 \%$ less new-onset diabetes. In the diabetic subset of this trial, conducted in 1195 patients, there was a statistically significant reduction in all-cause mortality and cardiovascular mortality. Reductions of stroke and myocardial infarction were also seen, but these trends were not statistically significant largely because of the small numbers.

It is fair to say that in the treatment of type II diabetes, there is very little evidence that antidiabetic treatment is associated with a reduction in mortality. The results of two trials that have been conducted in this area have been disappointing. Therefore, the LIFE trial is the first-ever study to show that any medical treatment is of value in reducing all-cause mortality in type II diabetes. There is evidence that the HMG CoA reductase inhibitors (the statins) reduce mortality in diabetic survivors from myocardial infarction and high-risk diabetics in general.

The results of the LIFE trial raise a great many questions. Why was losartan so much better than atenolol? It wasn't due to differences in blood pressure. The possibility therefore that blockade of the angiotensin receptor is beneficial in high-risk hypertensives needs to be investigated and the mechanisms will be the source of much research over the oncoming years.

The LIFE trial was conducted on an international basis in Scandinavia, the USA and the UK. There are several more subgroup analyses yet to be published and also many official LIFE trial substudies have been conducted. This special issue of the Journal of Human Hypertension is devoted to the publication of a series of these substudies. In general, it has not been the policy of this journal to endorse any one antihypertensive agent mainly because there was no reason to do so. We have broken our own rules on this occasion simply because the LIFE trial does provide ground breaking new data which will make us all have to rethink the way we manage our hypertensive patients.

Professor DG Beevers

The University Department of Medicine, City Hospital, Birmingham, UK 